\title{
Aneurisma de artéria ilíaca interna roto: relato de caso
}

\author{
Ruptured internal iliac artery aneurysm: case report \\ Cristina Toledo Afonso", Ricardo Jayme Procópio², Túlio Pinho Navarro ${ }^{3}$, \\ Gustavo Henrique Dumont Kleinsorge ${ }^{4}$, Beatriz Deoti e Silva Rodrigues ${ }^{5}$, \\ Marco Antônio Gonçalves Rodrigues ${ }^{6}$
}

\section{Resumo}

Aneurismas isolados da artéria ilíaca interna são raros, acometem $0,1 \%$ da população e correspondem a $1 \%$ dos aneurismas aorto-ilíacos. $\mathrm{Na}$ maioria das vezes, os pacientes são assintomáticos, mas podem apresentar dor abdominal, massa pulsátil no hipogástrio ou na fossa ilíaca, sintomas compressivos urinários, gastrointestinais ou neurológicos. Podem ocasionar quadro de abdome agudo, principalmente quando há ruptura. O diagnóstico precoce dos aneurismas isolados de artéria ilíaca interna é incomum, sendo identificados quando mais volumosos ou rotos, o que aumenta significativamente sua morbimortalidade e torna seu prognóstico mais reservado. Dessa forma, representam um desafio terapêutico. A ligadura cirúrgica tem sido o tratamento mais comum, entretanto a cirurgia endovascular tem mostrado bons resultados, inclusive nos aneurismas rotos. É relatado caso de aneurisma de artéria ilíaca interna isolado roto diagnosticado durante laparotomia para abordagem de abdome agudo.

Palavras-chave: Aneurisma roto, artéria ilíaca, abdome agudo.

\section{Introdução}

$\mathrm{O}$ aneurisma isolado da artéria ilíaca interna é condição incomum, grave e de difícil diagnóstico. Acomete cerca de $0,1 \%$ da população em geral e corresponde a aproximadamente $1 \%$ dos aneurismas aorto-ilíacos ${ }^{1-3}$, sendo a artéria ilíaca comum a mais envolvida (85\%), seguida da artéria ilíaca interna $(10 \%)$ e artéria ilíaca externa $(1 \%)^{4}$. Esse tipo de aneurisma é mais frequente em homens e idosos (média de 69 anos) ) $^{2-4}$. Cerca de $50 \%$ são bilaterais ${ }^{5}$.

\begin{abstract}
Isolated internal iliac artery aneurysms are rare. They affect $0.1 \%$ of the population, and account for $1 \%$ of aortoiliac aneurysms. Patients are mostly asymptomatic, yet they can have abdominal pain, pulsatile mass in the hypogastrium or iliac fossa, or urinary, gastrointestinal or neurological compressive symptoms. Such aneurysms are likely to course with an acute abdomen, especially when ruptured. Early diagnosis of isolated internal iliac artery aneurysms is difficult, as they are more easily detected when larger or ruptured, which significantly raises their morbidity and mortality rate and determines a poor prognosis. Therefore, they are a therapeutic challenge. Surgical ligation has been the most common treatment; however, the endovascular approach has presented good outcomes, even in the event of ruptured aneurysms. A case of ruptured isolated iliac artery aneurysm diagnosed during a laparotomy (acute abdomen approach) is reported.
\end{abstract}

Keywords: Ruptured aneurysm, iliac artery, acute abdomen.

$\mathrm{Na}$ maioria das vezes é assintomático, encontrando-se frequentemente roto nos quadros manifestos $^{6}$. Quando sintomático, os sinais e sintomas dependem do tamanho, localização e relação com estruturas adjacentes.

\section{Relato de caso}

Paciente do sexo masculino, 67 anos, com quadro súbito de dor abdominal, referida em região lombar e em fossa ilíaca direita, associada à disúria e constipação

1. Residente, Cirurgia Geral, Hospital das Clínicas, Universidade Federal de Minas Gerais (UFMG), Belo Horizonte, MG.

2. Coordenador, Departamento de Cirurgia Endovascular, Hospital das Clínicas, UFMG, Belo Horizonte, MG.

3. Coordenador, Departamento de Cirurgia Vascular, Hospital das Clínicas, UFMG, Belo Horizonte, MG.

4. Residente, Cirurgia Vascular, Hospital das Clínicas, UFMG, Belo Horizonte, MG.

5. Mestre, Cirurgia, UFMG, Belo Horizonte, MG. Membro, Grupo de Urgência e Proctologia, Instituto Alfa de Gastroenterologia, Hospital das Clínicas, UFMG, Belo Horizonte, MG.

6. Professor, Departamento de Cirurgia, Faculdade de Medicina, UFMG, Belo Horizonte, MG.

Não foram declarados conflitos de interesse associados à publicação deste artigo.

Artigo submetido em 04.08.08, aceito em 07.11.08. 
há 24 horas. Estado geral preservado, estabilidade hemodinâmica, oligúria, peristaltismo diminuído e massa endurecida palpável suprapúbica. Tabagista, hipertenso, tendo realizado angioplastia coronariana com stent há 2 anos.

Exames laboratoriais revelaram hemoglobina de 11,3 $\mathrm{g} / \mathrm{dL}$ e leucócitos de $14.500 / \mathrm{mm}^{3}$. A radiografia de abdome evidenciou distensão de alças, sem níveis hidroaéreos e a retossigmoidoscopia mostrou sinais de isquemia de mucosa. Realizou-se sondagem urinária de alívio sem progressão da sonda. O paciente evoluiu com piora da dor, aumento da massa, hipotensão e choque. Foi encaminhado ao bloco cirúrgico com suspeita de vôlvulo de sigmoide.

Durante a laparotomia, constatou-se hematoma extenso que se infiltrava pela bexiga, retroperitônio e musculatura anterior da parede abdominal. Realizou-se controle aórtico proximal e exploração do eixo aortoilíaco. Foi identificado aneurisma da artéria ilíaca interna direita roto, de $5 \mathrm{~cm}$ de diâmetro, com deslocamento anterior do ureter e compressão de bexiga e reto, sem outras formações aneurismáticas. Procedeu-se à abertura do saco aneurismático e à ligadura desse a suas origens proximal e distal (Figuras 1 e 2). Repuseram-se $900 \mathrm{~mL}$ de concentrado de hemácias e $600 \mathrm{~mL}$ de plasma. Foi encaminhado ao centro de terapia intensiva, onde evoluiu com estabilidade clínica. Recebeu alta para enfermaria no sexto dia pós-operatório e hospitalar no nono dia. Encontra-se em controle ambulatorial há 4 meses, assintomático e em boas condições clínicas.

\section{Discussão}

Os aneurismas isolados de artéria ilíaca apresentam desafios diagnósticos e terapêuticos. O fator etiológico mais comum é um processo degenerativo da parede vascular associado principalmente à aterosclerose ${ }^{4}$. Outras causas incluem infecção, trauma, iatrogenia, vasculites, doenças do colágeno e gravidez. Clinicamente, podem manifestar-se por meio de massa pulsátil, dor abdominal e/ou lombossacra, sintomas urinários, gastrointestinais ou neurológicos, além de fenômenos tromboembólicos ${ }^{6}$. Massa palpável pulsátil em fossa ilíaca ou ao toque vaginal e retal é encontrada em 55\% dos $\operatorname{casos}^{2}$. A dor pode manifestar-se de forma aguda,

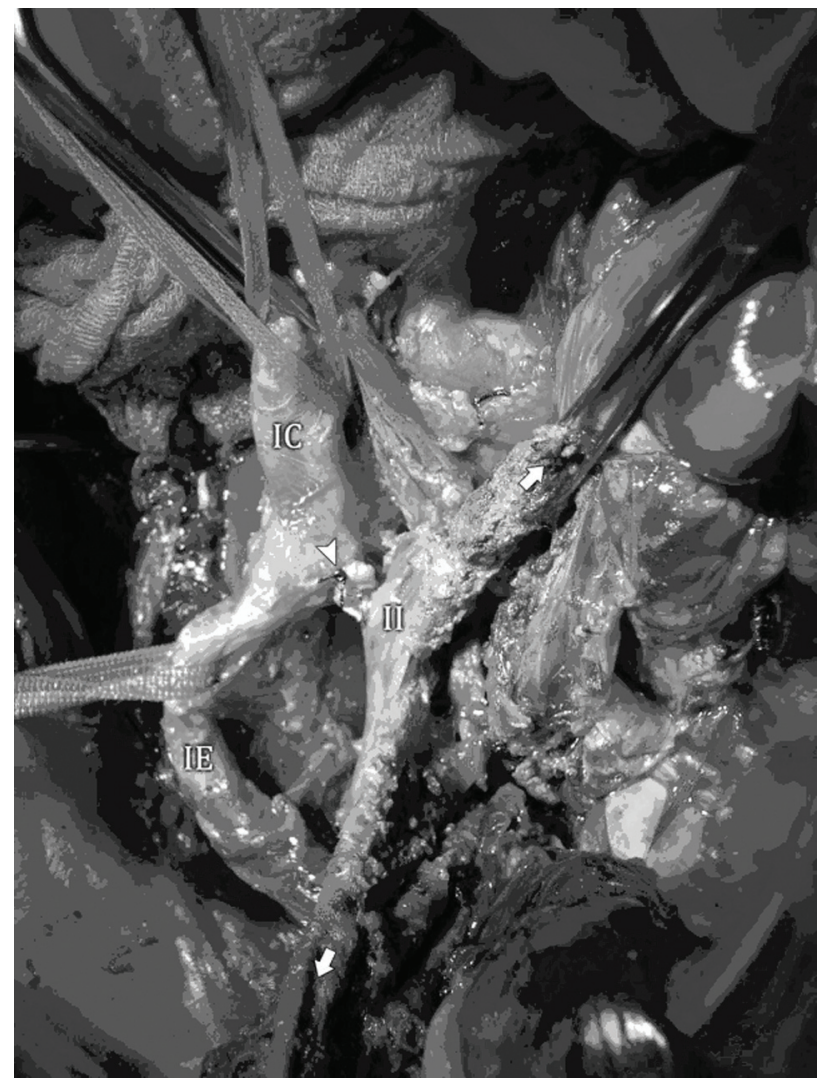

Figura 1 - Ligadura proximal (cabeça da seta) e distal da artéria ilíaca interna (II) com ressecção do saco aneurismático; artéria ilíaca comum (IC) e artéria ilíaca externa (IE)

por expansão ou ruptura, ou crônica, por compressão de nervos ou vísceras ${ }^{7,8}$. A compressão dos ureteres e da bexiga pode desencadear sintomas urinários $(54 \%$ dos casos $)^{9-11}$. As alças intestinais podem sofrer compressão levando a constipação, tenesmo, dor ao toque retal e enterorragia ${ }^{12,13}$. Trombose venosa profunda e embolia pulmonar podem advir da compressão do sistema ilíaco-femoral ${ }^{14,15}$.

Entretanto, na maioria dos casos, esses pacientes são assintomáticos até ocorrer ruptura. Clinicamente, a tríade clássica de ruptura de um aneurisma abdominal, composta por dor aguda e progressiva, hipotensão e massa pulsátil, está presente em 38 a 51\% dos pacientes. A ruptura pode ser retroperitoneal ou intraperitoneal, mas também para reto, ureter ou bexiga ${ }^{16,17}$. Quando há ruptura, a taxa de mortalidade nas cirurgias de urgência varia de 50 a $100 \%$, o que é significativamente maior quando comparada à taxa das cirurgias eletivas, que é 


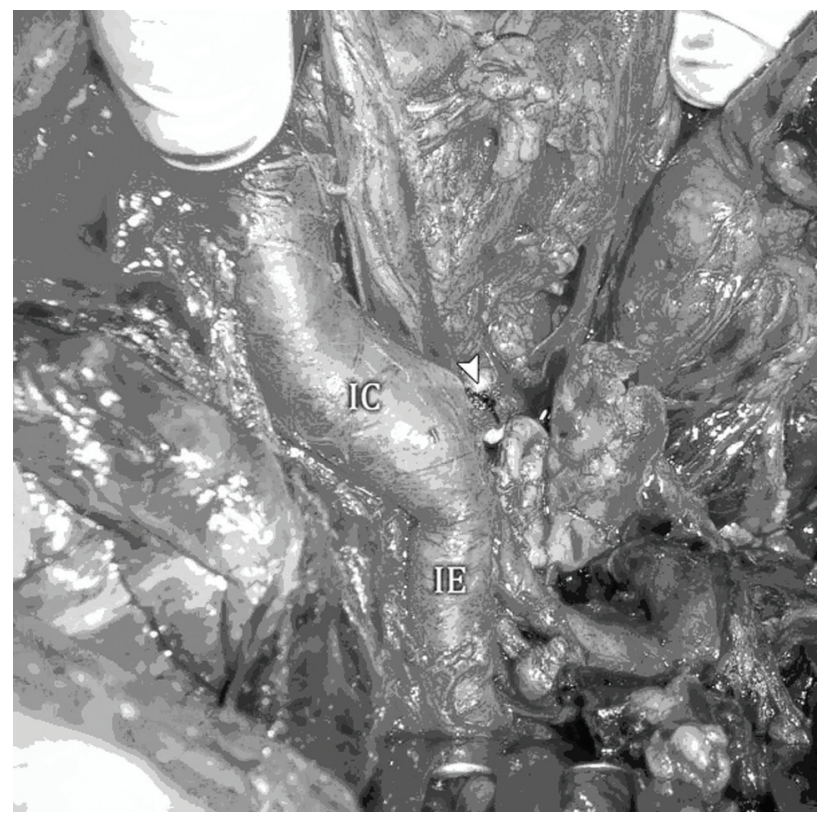

Figura 2 - Ligadura proximal (cabeça da seta) e distal da artéria ilíaca interna com ressecção do saco aneurismático; artéria ilíaca comum (IC) e artéria ilíaca externa (IE)

geralmente inferior a $10 \%^{18}$. No paciente em questão, a dor intensa, associada a sintomas compressivos de estruturas pélvicas, sinalizava para a afecção em estudo. $\mathrm{O}$ hematoma pélvico explica disúria, oligúria, constipação, distensão e sinais de sofrimento de alças intestinais.

O diagnóstico dos aneurismas isolados de artéria ilíaca interna, por sua localização profunda na pelve, dificilmente é realizado de forma precoce. Usualmente, são descobertos de forma incidental durante operações, exames periódicos, investigações de outras condições ou autópsia.

A ultrassonografia abdominal e pélvica é útil no diagnóstico, na triagem e seguimento dos pacientes assintomáticos ${ }^{6}$. A angiotomografia tem sido o padrão-ouro para o diagnóstico desse aneurisma ${ }^{19,20}$. É capaz de demonstrar localização, tamanho, tortuosidade, trajeto do aneurisma, relação com órgãos adjacentes, sinais de ruptura e hemorragia retroperitoneal ${ }^{21}$. Já a angiorressonância é método de exceção empregado em pacientes que não podem receber meio de contraste iodado. Atualmente, a angiografia é realizada somente na falha dos métodos prévios.
O diâmetro do aneurisma ilíaco influencia na indicação da intervenção: aqueles com diâmetros inferiores a $3 \mathrm{~cm}$ podem ser tratados conservadoramente, indicando-se intervenção em pacientes sintomáticos e/ou que apresentam aneurisma em expansão ou com diâmetro superior a $3 \mathrm{~cm}^{22,23}$.

O tratamento dos aneurismas isolados de artéria ilíaca interna representa um desafio devido à sua topografia na pelve, ao seu grande tamanho à época do diagnóstico e aos riscos oferecidos pela proximidade com importantes estruturas adjacentes. A ligadura cirúrgica tem sido o tratamento mais comumente empregado. $\mathrm{O}$ tratamento endovascular também vem sendo utilizado para os aneurismas ilíacos, inclusive de ilíaca interna isolada.

A ligadura do aneurisma pode ser apenas proximal ou combinada com ligadura distal, como no caso relatado. A ligadura proximal apresenta menor risco de sangramento por ser rápida. Porém, pode persistir o risco de ruptura por manutenção de fluxo intra-aneurismático retrógrado pelo segmento distal da artéria ou por colaterais, e não resolver os sintomas compressivos ${ }^{24}$. Por essa razão, a ligadura combinada é tratamento preferencial para a maioria dos pacientes.

A ressecção é técnica pouco utilizada devido a fibrose em torno do aneurisma, com risco aumentado de hemorragia ou dano a estruturas vizinhas, especialmente ureter e veias ilíacas, que estão frequentemente aderidos à sua parede ${ }^{6}$. Portanto, deve-se evitar a dissecção circunferencial das artérias ilíacas com o intuito de minimizar tais complicações ${ }^{25}$.

A utilização da cirurgia endovascular tem aumentado por apresentar vantagens, como permitir acesso por punção percutânea da artéria femoral, além de proporcionar menores traumas, hemorragias, necessidade de hemoderivados e permanência hospitalar ${ }^{26}$. Além disso, tal cirurgia não é influenciada por dificuldades de dissecção, como nos obesos e no abdome hostil. Alguns estudos compararam o tratamento endovascular com o convencional e demonstraram resultados semelhantes, com bons resultados nos aneurismas rotos ${ }^{27-30}$.

Não há consenso na literatura sobre como proceder nos casos de aneurismas bilaterais da artéria ilíaca inter$\mathrm{na}^{31,32}$. Entretanto, a preservação de pelo menos uma 
artéria é preconizada pela maioria dos autores, pois impotência sexual, isquemia colônica, vesical e da musculatura pélvica são complicações comuns associadas à ligadura bilateral dessas artérias. A correção cirúrgica através da interposição de enxertos é a modalidade terapêutica mais adequada em tal circunstância ${ }^{25,33}$.

\section{Conclusão}

Os aneurismas da artéria ilíaca interna são raros e, quando rotos, podem cursar com quadro de abdome agudo. Apresentam alta morbimortalidade e têm prognóstico bastante reservado, particularmente nos casos de ruptura. A ligadura cirúrgica do vaso, nessas situações, é a técnica mais utilizada para estancar a hemorragia e tratar o aneurisma, evitando-se extensas dissecções e encurtando o tempo operatório.

\section{Referências}

1. Olsen PS, Schroeder T, Agerskov K, et al. Surgery for abdominal aortic aneurysms. A survey of 656 patients. J Cardiovasc Surg. 1991;32:636-42.

2. Richardson JW, Greenfield LJ. Natural history and management of iliac aneurysms. J Vasc Surg. 1988;8:165-71.

3. Schuler JJ, Flanigan DP. Iliac artery aneurysms. In: Bergan JJ, Yao JST, editors. Aneurysms, diagnosis and treatment. New York: Grune \& Stratton; 1982. p. 469-85.

4. McCready RA, Pairolero PC, Gilmore JC, Kazmier FJ, Cherry KJ, Hollier LH. Isolated iliac artery aneurysms. Surgery. 1983;93:688-93.

5. Krupski WC, Selzman CH, Floridia R, Strecker PK, Nehler MR, Whitehill TA. Contemporary management of isolated iliac aneurysms. J Vasc Surg. 1998;28:1-11.

6. Dix FP, Titi M, Al-Khaffaf $\mathrm{H}$. The isolated internal iliac artery aneurysm: a review. Eur J Vasc Endovasc Surg. 2005;30:119-29.

7. Chapman EM, Shaw RS, Kubik CS. Sciatic pain from arteriosclerotic aneurysm of pelvic arteries. N Engl J Med. 1964;271:1410-1.

8. Wider C, Kuntzer T, Von Segesser LK, Qanadli SD, Bogousslavsky J, Vingerhoets F. Bilateral compressive lumbosacral plexopathy due to internal iliac artery aneurysms. J Neurol. 2006;253:809-10.

9. Honma I, Takagi Y, Shigyo M, Sunaoshi K, Inaoka M, Miyao N. Massive hematuria after cystoscopy in a patient with an internal iliac artery aneurysm. Int J Urol. 2002;9:407-9.

10. Mineta K, Nomura M, Fujimoto N, Takahashi K, Matsumoto T. Ureteral obstruction due to retroperitoneal fibrosis secondary to a solitary internal iliac aneurysm. Int $\mathbf{J}$ Urol. 2004;11:1024-27.

11. O'Driscoll D, Fitzgerald E. Isolated iliac artery aneurysms with associated hydronephrosis. J R Coll Surg Edinb. 1999;44:197-9.

12. Wu C, Nguyen BD, Regan F. An internal iliac artery aneurysm masquerading as a rectal mass. Clin Radiol. 2000;55:319-20.
13. Ozergin U, Vatansev C, Durgut K, Ozulku M, Gormus N. An internal iliac artery aneurysm causing a colonic obstruction: report of a case. Surg Today. 2001;31:839-41.

14. Rosenthal D, Matsuura JH, Jerius H, Clark MD. Iliofemoral venous thrombosis caused by compression of an internal iliac artery aneurysm: a minimally invasive treatment. J Endovasc Surg. 1998;5:142-5.

15. Su WT, Goldman KA, Riles TS, Rosen R. Deep venous thrombosis with pulmonary embolus after selective embolization of an internal iliac artery aneurysm. J Vasc Surg. 1996;23:152-5.

16. Jackman RJ, McGuarrie HB, Edwards JE. Fatal hemorrhage caused by aneurysm of the internal iliac artery: report of a case. Proc Mayo Clinic. 1948;23:305.

17. De Donato G, Neri E, Baldi I, Setacci C. Rupture of internal iliac artery aneurysm presenting as rectus sheath hematoma: case report. J Vasc Surg. 2004;39:250-3.

18. Boyarsky AH, Burks WP, Davidson JT, Chandler JJ. Ruptured aneurysm of the internal iliac artery. South Med J. 1985;78:1356-7.

19. Raptopoulos V, Rosen MP, Kent KC, Kuestner LM, Sheiman RG, Pearlman JD. Sequential helical CT angiography of aortoiliac disease. AJR Am J Roentgenol. 1996;166:1347-54.

20. Rieker O, Duber C, Neufang A, Pitton M, Schweden F, Thelen M. CT angiography versus intraarterial digital subtraction angiography for assessment of aortoiliac occlusive disease. AJR Am J Roentgenol. 1997;169:1133-8.

21. Todd GJ, Nowygrod R, Benvenisty A, Buda J, Reemtsma K. The accuracy of CT scanning in the diagnosis of abdominal and thoracoabdominal aortic aneurysms. J Vasc Surg. 1991;13:302-10.

22. Santilli SM, Wernsing SE, Lee ES. Expansion rates and outcomes for iliac artery aneurysms. J Vasc Surg. 2000;31:114-21.

23. Owen RJ, Jackson R, Loose HW, Lees TA, Dunlop P, Rose JD. Percutaneous ablation of an internal iliac aneurysm using tissue adhesive. Cardiovasc Intervent Radiol. 2000;23:389-91.

24. Ruurda JP, Rijbroek A, Vermeulen EG, Wisselink W, Rauwerda JA. Continuing expansion of internal iliac artery aneurysms after surgical exclusion of the inflow. A report of two cases. J Cardiovasc Surg. 2001;42:389-92.

25. Carvalho ATY, Prado V, Neto HJG, Caffaro RA. Aspectos cirúrgicos dos aneurismas isolados das artérias ilíacas. J Vasc Bras. 2006;5:203-8.

26. Chaer RA, Barbato JE, Lin SC, Zenati M, Kent KC, McKinsey JF. Isolated iliac artery aneurysms: a contemporary comparison of endovascular and open repair. J Vasc Surg. 2008;47:708-13.

27. Van Kelckhoven BJ, Bruijninckx BM, Knippenberg B, Van Overhagen $\mathrm{H}$. Ruptured internal iliac artery aneurysm: staged emergency endovascular treatment in the interventional radiology suite. Cardiovasc Intervent Radiol. 2007;30:774-7.

28. Ricci MA, Najarian K, Healey CT. Successful endovascular treatment of a ruptured internal iliac aneurysm. J Vasc Surg. 2002;35:1274-6. 
29. Silva AAM, Reis LF, Miquelin DG, Sano PY, Pereira VS, Godoy JMP. Tratamento endovascular de aneurisma de ilíaca roto: relato de caso. J Vasc Bras. 2007;6:78-81.

30. Lobato AC, Almeida FHM. Aneurismas periféricos: tratamento endovascular. In: Brito CJ, Duque AC, Merlo I, Silva RM, Filho VLF, organizadores. Cirurgia vascular, cirurgia endovascular e angiologia. $2^{\mathrm{a}}$ ed. Rio de Janeiro: Revinter; 2008. p. 1339-50.

31. Karch LA, Hodgson KJ, Mattos MA, Bohannon WT, Ramsey DE, McLafferty RB. Adverse consequences of internal iliac artery occlusion during endovascular repair of abdominal aortic aneurysms. J Vasc Surg. 2000;32:676-83.

32. Bratby MJ, Munneke GM, Belli AM, et al. How safe is bilateral internal iliac artery embolization prior to EVAR? Cardiovasc Intervent Radiol. 2008;31:246-53.
33. Faries PL, Morrissey N, Burks JA, et al. Internal iliac artery revascularization as an adjunct to endovascular repair of aortoiliac aneurysms. J Vasc Surg. 2001;34:892-9.

Correspondência:

Cristina Toledo Afonso

Rua Professor Antônio Aleixo, 760/1501,

Bairro Lourdes

CEP 30180150 - Belo Horizonte, MG

Tel.: (31) 3337.9836, (31) 9321.9676

E-mail: cristinatafonso@yahoo.com.br

\section{Mudança no endereço eletrônico da SBACV Nacional}

Prezado Associado:

O endereço do site da SBACV Nacional foi alterado para:

www.sbacv.com.br

O domínio dos e-mails também mudou:

@sbacv.com.br

Atualize hoje mesmo os endereços da SBACV Nacional no seu navegador e gerenciador de emails. 